\title{
Crystal nucleation in silicate glasses: the temperature and size dependence of crystal/liquid surface energy
}

\author{
Vladimir M. Fokin ${ }^{1}$, Edgar D. Zanotto * \\ LaMaV-Vitreous Materials Laboratory, DEMa - Department of Materials Engineering, Ufscar - Federal University of São Carlos, \\ 13.565-905, São Carlos - SP, Brazil
}

Received 28 June 1999; received in revised form 29 September 1999

\begin{abstract}
The most basic assumption of the classical nucleation theory (CNT) is to treat nucleus/liquid surface energy, $\sigma$, as a macroscopic property having a value equal to that of a planar interface, $\sigma_{\infty}$. Therefore, when the CNT is used to analyze experimental data, the size dependence of surface energy is often neglected. To date, there has been no reliable method to measure the surface energy of the nucleus/liquid interface except by fitting nucleation rate data to the theory. In this case, one obtains the surface energy of critical size nuclei as a function of temperature. However, the fitted $\sigma(T)$ dependence arises from two different factors: the temperature dependence of $\sigma$ for a planar interface and its size dependence. This paper focuses on the temperature dependence of the macroscopic value of surface energy, decoupling it from the size dependent part. Tolman's equation was used to eliminate the size dependence of surface energy from published nucleation data for two stoichiometric silicate glasses $\left(\mathrm{Li}_{2} \mathrm{O} \cdot 2 \mathrm{SiO}_{2}\right.$ and $\left.\mathrm{Na}_{2} \mathrm{O} \cdot 2 \mathrm{CaO} \cdot 3 \mathrm{SiO}_{2}\right)$. It is shown that the Tolman parameter may be chosen so that surface tension decreases with temperature; $\mathrm{d} \sigma_{\infty} / \mathrm{d} T<0$. The value of $\mathrm{d} \sigma_{\infty} / \mathrm{d} T$ obtained in this way is close to theoretical predictions. (C) 2000 Elsevier Science B.V. All rights reserved.
\end{abstract}

\section{Introduction}

According to the classical nucleation theory (CNT) the nucleus/liquid interfacial energy, $\sigma$, governs the crystal nucleation rate to a large extent [1]. The most serious assumption of the CNT, however, is that the thermodynamic properties of nanometric aggregates of the newly nucleated phase are the same as those of the corresponding bulk phase. Thus, nucleus/liquid surface energy is

\footnotetext{
${ }^{*}$ Corresponding author. Tel:: +55-16 260 8250; fax: +55-16 2615404.

E-mail address: dedz@power.ufscar.br (E.D. Zanotto).

${ }^{1}$ On sabbatical leave from the Institute of Silicate Chemistry, Odoevskogo 24/2, 199155 St. Petersburg, Russia.
}

treated as a macroscopic property with a value equal to the respective value for a planar interface, $\sigma_{\infty}$. This assumption is known as the capillarity approximation.

Due to the lack of a reliable (direct) method to measure the surface energy of the nucleus/liquid interface, independently of nucleation experiments, one is forced to fit experimental data to theory. Hence, $\sigma_{\infty}$ cannot be compared directly with experimental values.

When the CNT is used to treat experimental data, not only the size dependence of surface energy but also its temperature dependence is often neglected. The latter approximation allows for the calculation of $\sigma_{\infty}$ and the pre-exponential term, $A_{\text {exp }}$, of the nucleation rate equation from experimental nucleation rate data at various 
temperatures. However, large discrepancies between the experimental pre-exponential term, $A_{\exp }$ and those predicted by the CNT, $A_{\text {theor }}$, were observed, e.g., [2,3]. However, it should be stressed that $A_{\text {exp }}$ is very sensitive to the choice of the thermodynamic driving force for crystallization (the difference between the volume free energies of glass and crystal). Nevertheless, in every case, $A_{\text {exp }}$ is higher than $A_{\text {theor }}$ and the discrepancy may reach $\sim 130$ orders of magnitude.

Such a discrepancy can be avoided by calculating a specific surface energy from experimental nucleation rate data at each temperature, using the theoretical value of the pre-exponential term. Consequently, surface energy may be used as a fit parameter and its apparent temperature dependence can thus be obtained. This approach was first applied to the study of metallic systems [4,5] and then to silicate glasses [6,7]. Surface energy, calculated in such a way, slightly increases with temperature $\left(\mathrm{d} \sigma / \mathrm{d} T \sim(0.06-0.16) \times 10^{-3} \mathrm{~J} \mathrm{~m}^{-2} \mathrm{~K}^{-1}\right)$. For instance, $\mathrm{d} \sigma / \mathrm{d} T=0.059 \times 10^{-3}$ for $\mathrm{Ga}$ [4], $0.09 \times 10^{-3}$ for $\mathrm{Hg}$ [5], $0.16 \times 10^{-3}$ [6], $0.13 \times 10^{-3}$ for L2S [7], $0.114 \times 10^{-3}$ [3], $0.128 \times 10^{-3}$ for $\mathrm{N} 2 \mathrm{C} 3 \mathrm{~S}$ [8], $0.129 \times 10^{-3} \mathrm{~J} \mathrm{~m}^{-2} \mathrm{~K}^{-1}$ [this work] for 2NC3S; where L2S, N2C3S and 2NC3S denote glasses of stoichiometric composition $\mathrm{Li}_{2} \mathrm{O} \cdot 2 \mathrm{SiO}_{2}$, $\mathrm{Na}_{2} \mathrm{O} \cdot 2 \mathrm{CaO} \cdot 3 \mathrm{SiO}_{2}$ and $2 \mathrm{Na}_{2} \mathrm{O} \cdot \mathrm{CaO} \cdot 3 \mathrm{SiO}_{2}$.

Since all thermodynamic properties are temperature dependent, it is reasonable to assume that this also applies to surface energy. The temperature dependence of $\sigma_{\infty}$ is also predicted by theory, see e.g., [9]. In particular, it has been shown [10] that $\mathrm{d} \sigma_{\infty} / \mathrm{d} T<0$ when the molar volume of the liquid phase is higher than the molar volume of the crystal phase. A similar proposal was advanced in [11], i.e., crystal-melt surface energy should significantly increase with decreasing temperature, starting from the thermodynamic crystallization temperature. At temperatures below glass transition, $\sigma_{\infty}$ could be almost as large as the surface energy of high-angle grain boundaries of the corresponding crystalline solid.

Moreover, there is experimental evidence that the liquid-gas surface tension of some glassforming silicate melts decreases with increasing temperature [12]. According to [13], liquid-gas surface tension and crystal-liquid surface energy are directly proportional to each other. Hence, one may expect that the latter also decreases with increasing temperature, contrary to the experimental results using the procedure described previously, i.e., fitting nucleation rate data to the CNT.

It should be stressed again that the surface energy $\sigma(T, R)$, calculated from nucleation data, refers to nuclei of critical size, $R^{*}$. The latter parameter, in turn, changes with temperature. Hence, as noted in [14], a $\sigma(T)$ plot may partially reflect a size effect. The issue of the size dependence of surface energy was elaborated in a number of papers, e.g., Refs. [14-17].

This paper focuses on the temperature dependence of the specific macroscopic surface energy, decoupling it from the size dependent part. Our aim is to calculate the surface energy of the crystalmelt interface as a function of temperature, $\mathrm{d} \sigma_{\infty} / \mathrm{d} T$, using crystal nucleation data for two silicate glasses and to compare it with theoretical predictions.

\section{Theoretical background}

According to the classical nucleation theory, e.g., [1], the homogeneous nucleation rate, $I_{\text {hom, }}$, can be written as

$I_{\text {hom }}=A_{\exp }\left(-\frac{\Delta G_{\mathrm{D}}+W^{*}}{k T}\right)$,

$A=2 N_{1} v_{0}\left(\frac{\sigma a^{2}}{k T}\right)^{1 / 2}$,

where $A$ is the pre-exponential term, $k$ is Boltzmann's constant, $N_{1} \cong 1 / a^{3}$ is the number of structural (formula) units of melt with size $a$ per unit volume, $v_{0}=k T / h$ is the vibration frequency of a structural unit (for typical nucleation temperatures, $v_{0} \cong 10^{13} \mathrm{~s}^{-1}$ ), $h$ is Planck's constant, $\sigma$ is the nucleus/melt free energy per unit area, $\Delta G_{\mathrm{D}}$ is the activation energy for transport of a structural unit across the nucleus/melt interface and $W^{*}$ is the work of forming a critical nucleus.

From Eq. (1), the work of forming a critical nucleus (or the Gibbs free energy change due to the nucleus formation) determines, to a large 
degree, the value of nucleation rate. It is wellknown that if one neglects the strain energy associated to the formation of nuclei of size $R$ (for simplicity, we assume nuclei of spherical shape) $W$ can be written as

$W=\frac{4}{3} \pi R^{3} \Delta G_{\mathrm{V}}-4 \pi R^{2} \sigma$,

where $\Delta G_{\mathrm{V}}$ is the difference between the free energies of glass and crystal per unit volume of crystal. Fig. 1 shows the change of the Gibbs free energy as a function of the nucleus size for different approximations using typical values of the parameters given in the figure caption. The full curve (1) corresponds to the assumption of constant surface energy, $\sigma$ and constant crystal density, $\rho$. However, as mentioned earlier, such assumptions may be incorrect in the case of nanometric size clusters. For example, regarding the description of a nucleus, Rusanov [9] claims that "A revision of the nucleation theories is required, considering the size dependence of the surface tension and of other thermodynamic parameters". Thus, an account of the curvature dependence of the surface tension may result in significant

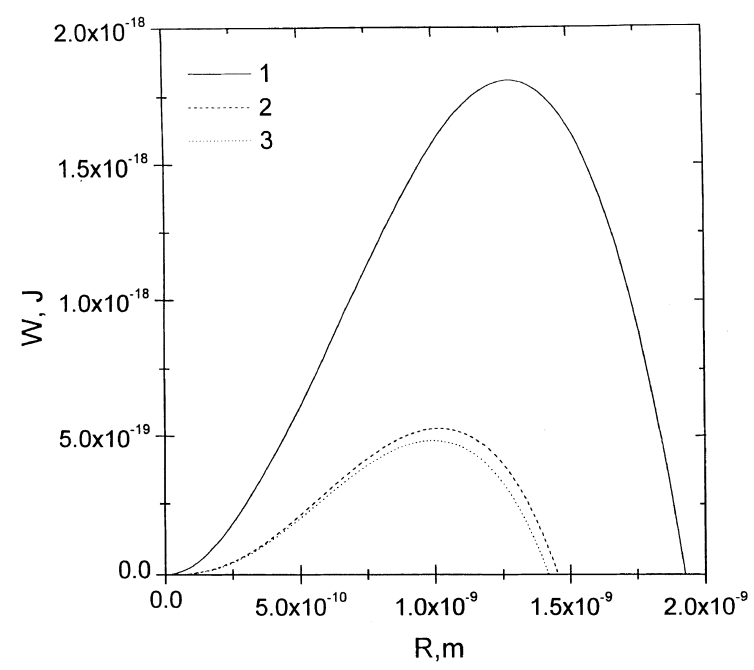

Fig. 1. Free energy change of the system due to the formation of a nucleus as a function of size. $1-\sigma$ and $\rho$ are size independent; $2-\sigma$ is size dependent (Eq. (4)); $3-\sigma$ and $\rho$ are size dependent. The following parameters were used in the calculations: $2 \delta=4.7 \times 10^{-10} \mathrm{~m}, \Delta G_{\mathrm{V}}=4.08 \times 10^{8} \mathrm{~J} \mathrm{~m}^{-3}$, $K=5 \times 10^{-11} \mathrm{~m}^{2} \mathrm{~N}^{-1}, \sigma=0.262 \mathrm{~J} \mathrm{~m}^{-2}$. quantitative changes in the work of critical cluster formation.

There have even been suggestions that an account of this effect may result in a qualitative change of the shape of the thermodynamic barrier for nucleation, $W^{*}[18]$. A critical discussion of this point of view was given in [16].

Several approximate equations have been suggested for the curvature dependence of the surface energy, $\sigma=\sigma(R)$, e.g., [19-22]

$\sigma(R)=\sigma_{\infty} \exp (-2 \delta / R), \quad$ Ref. [19] Gibbs,

$\sigma(R)=\sigma_{\infty} /(1+2 \delta / R), \quad$ Ref. [20] Tolman,

$\sigma(R)=\sigma_{\infty}(1-\delta / R)^{2}, \quad$ Ref. [21] Rasmussen,

$\sigma(R)=\sigma_{\infty}(1-2 \delta / R), \quad$ Ref. [22] Vogelsberger,

where $\sigma_{\infty}$ is the surface tension of a planar interface. Tolman's parameter, $\delta$, characterizes the width of the interfacial region between the coexisting phases (it has the order of atomic dimensions). A general equation for curvature dependent surface tension was derived in [23], which includes Eqs. (3)-(6) as particular cases. Using an analysis of the limits of their validity, given in [16], we have chosen Tolman's Eq. (4) because it encompasses the smallest size $(R)$ from which the equations are meaningful. Eq. (4) was derived for a liquid drop, assuming that $R \gg \delta$. It follows from our calculations (presented below) that the critical radius varies within a range of $1.7<R^{*} / \delta<4.6$. For this size range, Eq. (4) overestimates the surface tension by about $10-2.5 \%$, respectively [20].

The change of the system's free energy due to nucleus formation, when a size dependence of surface energy is taken into account, is shown in Fig. 1 by a dashed curve (2). Here, Eq. (4) is used for a description of $\sigma(R)$.

Strictly speaking, the size dependence of the crystal density $\left[\rho(R) \cong \rho_{\infty}+\rho_{\infty} \kappa 2 \sigma / R\right.$, where $\kappa$ is the isothermal compressibility and $\rho_{\infty}$ is the crystal 
density for $R \rightarrow \infty$ ] should also be taken into account [16]. For typical values of the thermodynamic parameters $\left(\kappa=5 \times 10^{-6} \mathrm{~atm}^{-1}\right.$, critical radius $R^{*}=10^{-9} \mathrm{~m}$ and $\sigma_{\infty}=0.2 \mathrm{~J} \mathrm{~m}^{-2}$ ), the ratio between the thermodynamic driving force per unit volume of critical crystal and that of a macroscopic crystal, $\Delta G_{\mathrm{V}}\left(R=R^{*}\right) / \Delta G_{\mathrm{V}}(R \rightarrow \infty)=$ $1+\kappa 2 \sigma_{\infty} / R^{*}$, is only 1.013 . On the other hand, if the crystal size varies from macroscopic to critical, the decreased surface tension leads to a decrease of the thermodynamic barrier of over three times (see Fig. 1, curves 1 and 2). The dotted curve (3) in Fig. 1 corresponds to the case where $\sigma$ and $\rho$ are size dependent. Thus, the use of a size dependent crystal density weakly affects the thermodynamic barrier of nucleation (compare curves 2 and 3). Hence, to a first approximation, we may treat the density of the nucleus as size independent.

One could also take into account the decrease of crystal density resulting from thermal expansion. Using the thermal expansion coefficient of polycrystalline lithium disilicate, $\alpha=1.205 \times 10^{-5}$ $\left(470-490^{\circ} \mathrm{C}\right)$ [24], the decrease in crystal density in the relevant temperature range for nucleation $\left(T_{\mathrm{g}}\right.$ to $T_{\mathrm{g}}+100^{\circ} \mathrm{C}$, where $T_{\mathrm{g}} \sim 450^{\circ} \mathrm{C}$ is the glass transition temperature) was estimated to be only $0.3 \%$. Thus, we assume that the $\rho(T)$ dependence is too weak to affect the $\sigma(T)$ calculated from nucleation data.

Expressing the kinetic barrier of nucleation, $\Delta G_{\mathrm{D}}$, in terms of the induction period of nucleation, $t_{\text {ind }}$, or of the viscosity, $\eta$, Eq. (1) may be transformed into Eq. (7) or Eq. (8), respectively, see e.g., $[8,25]$.

$T_{\mathrm{st}}=\frac{16}{3} \pi N_{1}^{2} \frac{(k T)^{1 / 2} \sigma^{3 / 2}}{\Delta G_{\mathrm{V}}^{2} t_{\text {ind }}} \exp \left(-\frac{W^{*}}{k T}\right)$,

$I_{\mathrm{st}}=2 N_{1}\left(\frac{\sigma a^{2}}{k T}\right)^{1 / 2} \frac{k T}{l^{3} \eta} \exp \left(-\frac{W^{*}}{k T}\right)$

where $I_{\mathrm{st}}$ is the steady-state homogeneous nucleation rate and $l$ has the order of $\mathrm{Si}-\mathrm{O}$ length.

The combination of Eqs. (2) and (4) results in

$W=\frac{4}{3} \pi R^{3} \Delta G_{\mathrm{V}}-4 \pi \frac{R^{3} \sigma_{\infty}}{R+2 \delta}$.

From the condition $(\partial W / \partial R)_{R^{*}}=0$ and taking the positive root one can find the critical radius $R^{*}$
$R^{*}=\frac{\left(\sigma_{\infty}-2 \Delta G_{\mathrm{V}} \delta\right)+\left(\sigma_{\infty}^{2}+2 \Delta G_{\mathrm{V}} \delta \sigma_{\infty}\right)^{1 / 2}}{\Delta G_{\mathrm{V}}}$

and then calculate the value of $W^{*}=W\left(R^{*}\right)$. Thus, on the right-hand side of Eqs. (9) and (10) and, correspondingly, of Eqs. (7) and (8), two parameters, crystal/liquid surface energy, $\sigma_{\infty}$ and Tolman's parameter, $\delta$, are unknown. The next part of the paper presents the results of fitting $\sigma_{\infty}$ to experimental nucleation data for different (fixed) values of $\delta$.

\section{Calculations and results}

Experimental nucleation rate data, induction times, viscosity and $\Delta G_{\mathrm{V}}$ for $\mathrm{Li}_{2} \mathrm{O} \cdot 2 \mathrm{SiO}_{2}$ and $\mathrm{Na}_{2} \mathrm{O} \cdot 2 \mathrm{CaO} \cdot 3 \mathrm{SiO}_{2}$ glasses from $[7,8,26,27]$ were used. These glasses exhibit internal, presumably homogeneous nucleation. Fig. 2 presents the temperature dependency of their steady-state
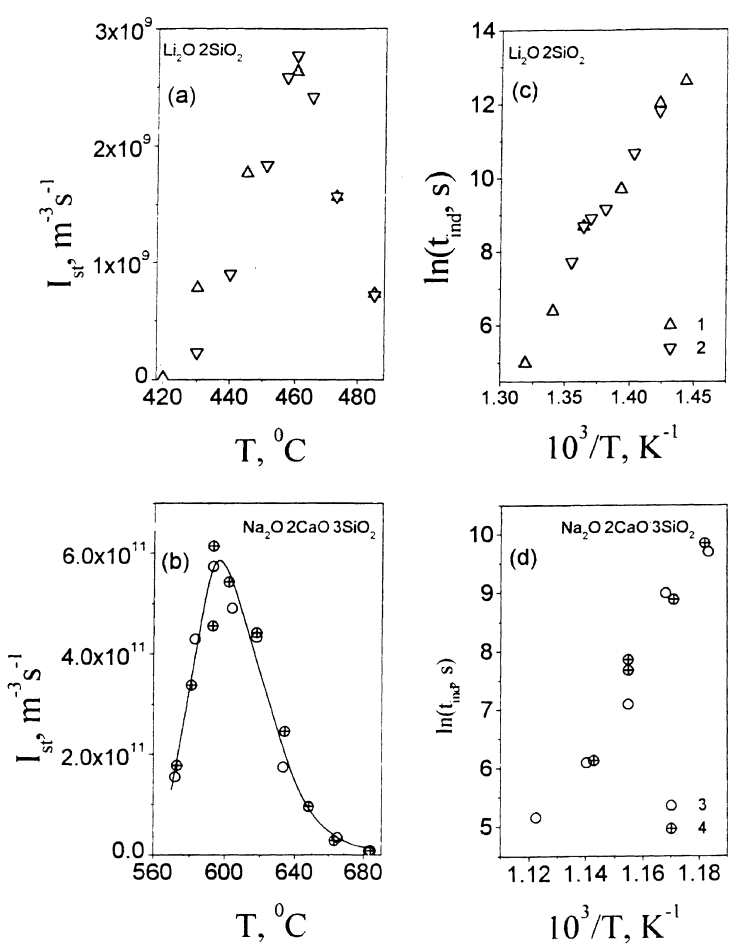

Fig. 2. Steady-state nucleation rates $(\mathrm{a}, \mathrm{b})$ and induction times for nucleation $(c, d)$ as a function of temperature for L2S (a, c) and $\mathrm{N} 2 \mathrm{C} 3 \mathrm{~S}(\mathrm{~b}, \mathrm{~d})$ glasses. The data were taken from the following references: 1 - [26], 2 - [7], 3 - [8], 4 - [27]. 
nucleation rates $(\mathrm{a}, \mathrm{b})$ and induction times $(\mathrm{c}, \mathrm{d})$ vs. reciprocal temperature plots.

The values of $I_{\text {st }}$ and $t_{\text {ind }}$, taken from the smoothed curves $I_{\text {st }}(T)$ (Fig. 2(a) and (b)) and from the Arrhenius plots $\ln \left(t_{\text {ind }}\right) \sim 1 / T$ (Fig. 2(c) and $(\mathrm{d}))$, were used for the calculations.

In the case of the $\mathrm{N} 2 \mathrm{C} 3 \mathrm{~S}$ glass, $\Delta G_{\mathrm{V}}$ was estimated by

$$
\begin{aligned}
\Delta G= & -\frac{\Delta H_{\mathrm{m}}}{T_{\mathrm{m}}}\left(T_{\mathrm{m}}-T\right)-\int_{T}^{T_{\mathrm{m}}} \Delta C_{\mathrm{p}} \mathrm{d} T^{\prime} \\
& +T \int_{T}^{T_{\mathrm{m}}} \frac{\Delta C_{\mathrm{p}}}{T^{\prime}} \mathrm{d} T^{\prime},
\end{aligned}
$$

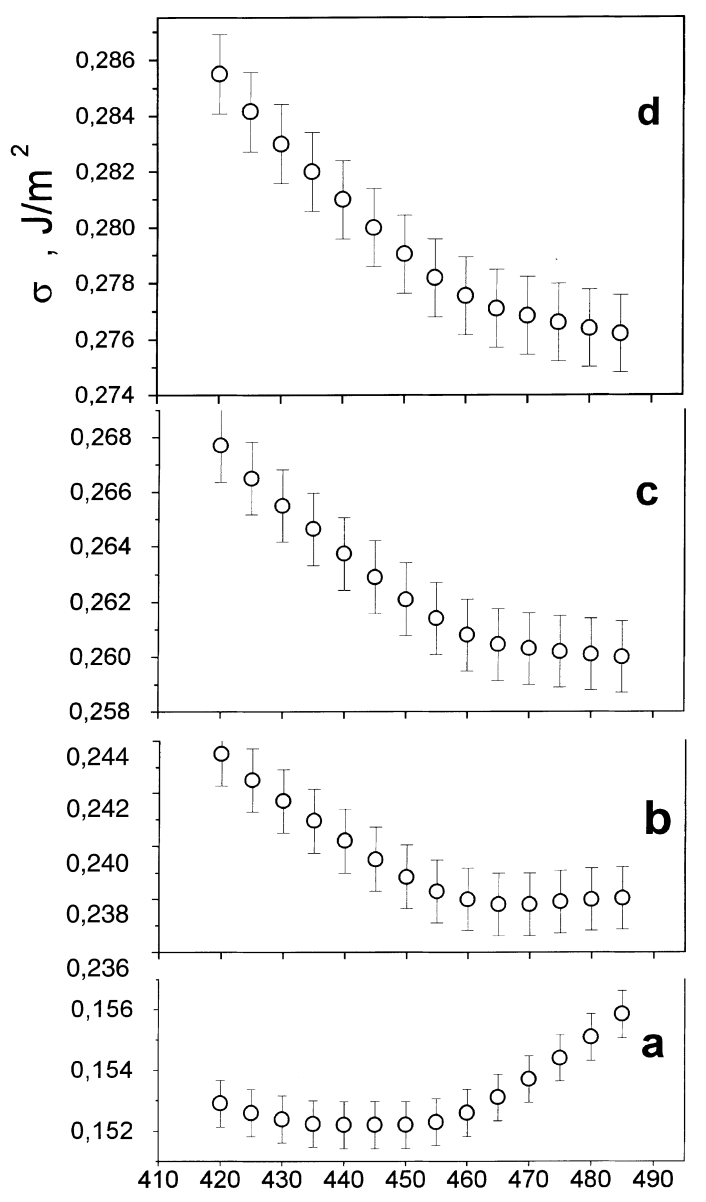

(A)

$\mathrm{T},{ }^{\circ} \mathrm{C}$ where $\Delta G_{\mathrm{V}}=-\Delta G / M, \Delta H_{\mathrm{m}}$ is the latent heat of melting per mole and $T_{\mathrm{m}}$ is the melting point, $\Delta C_{\mathrm{p}}=C_{\mathrm{p}}^{C}-C_{\mathrm{p}}^{L}(<0)$ is the difference in specific heats between the crystalline and liquid phases and $M$ is the molecular weight.

For the L2S glass, $\Delta G_{\mathrm{V}}\left(\mathrm{J} \mathrm{m}^{3}\right)=8.431 \times 10^{8}$ $-548258.655 T-73.00247 T^{2}[T(\mathrm{~K})]$ was used. This expression fits the experimental data [28] well in the temperature range 693-758 K. Thermodynamic and viscosity data were taken from $[29,30]$. The following Fulcher equation was employed to describe the viscosity of L2S glass: $\log (\eta, \mathrm{Pa} \mathrm{s})=$ $-7.52+6259 /(T-406), T(\mathrm{~K})$.

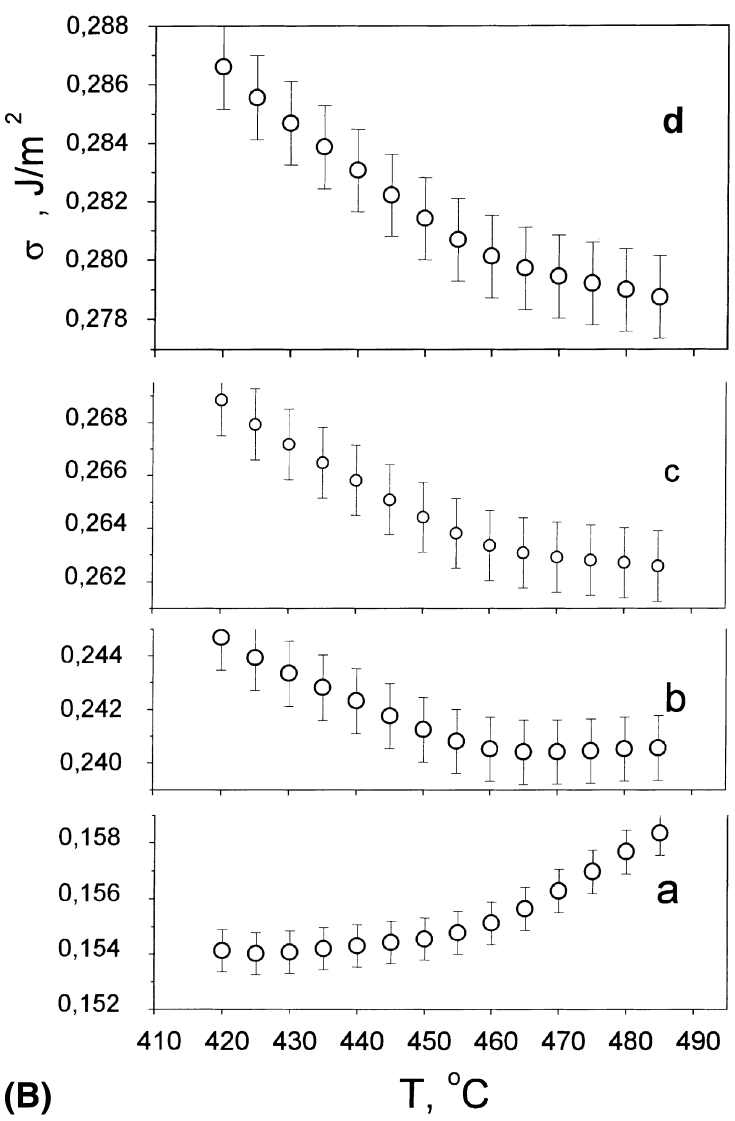

Fig. 3. (A) Crystal/ liquid surface energy for L2S glass calculated from Eq. (7) with different Tolman's parameters $\delta: 0$ (a); $2.33 \times 10^{-10}$ (b) $3 \times 10^{-10}$ (c) and $3.5 \times 10^{-10} \mathrm{~m}$ (d). (B) Crystal/ liquid surface energy for L2S glass calculated from Eq. (8) with different Tolman's parameters $\delta$ : $0(\mathrm{a}) ; 2.33 \times 10^{-10}(\mathrm{~b}) ; 3 \times 10^{-10}(\mathrm{c})$ and $3.5 \times 10^{-10} \mathrm{~m}(\mathrm{~d})$. 
A commercial software was used for the numerical solution of Eqs. (4), (7) and (8) to obtain $\sigma_{\infty}$ at different temperatures, using fixed values of Tolman's parameter, $\delta$. Figs. 3 and 4 show the $\sigma_{\infty}(T)$ plots for L2S and N2C3S glasses, respectively. For those values of $\sigma_{\infty}$, the ratio between critical radius and Tolman's parameter ranges from $1.7<R^{*} / \delta<4.6$.

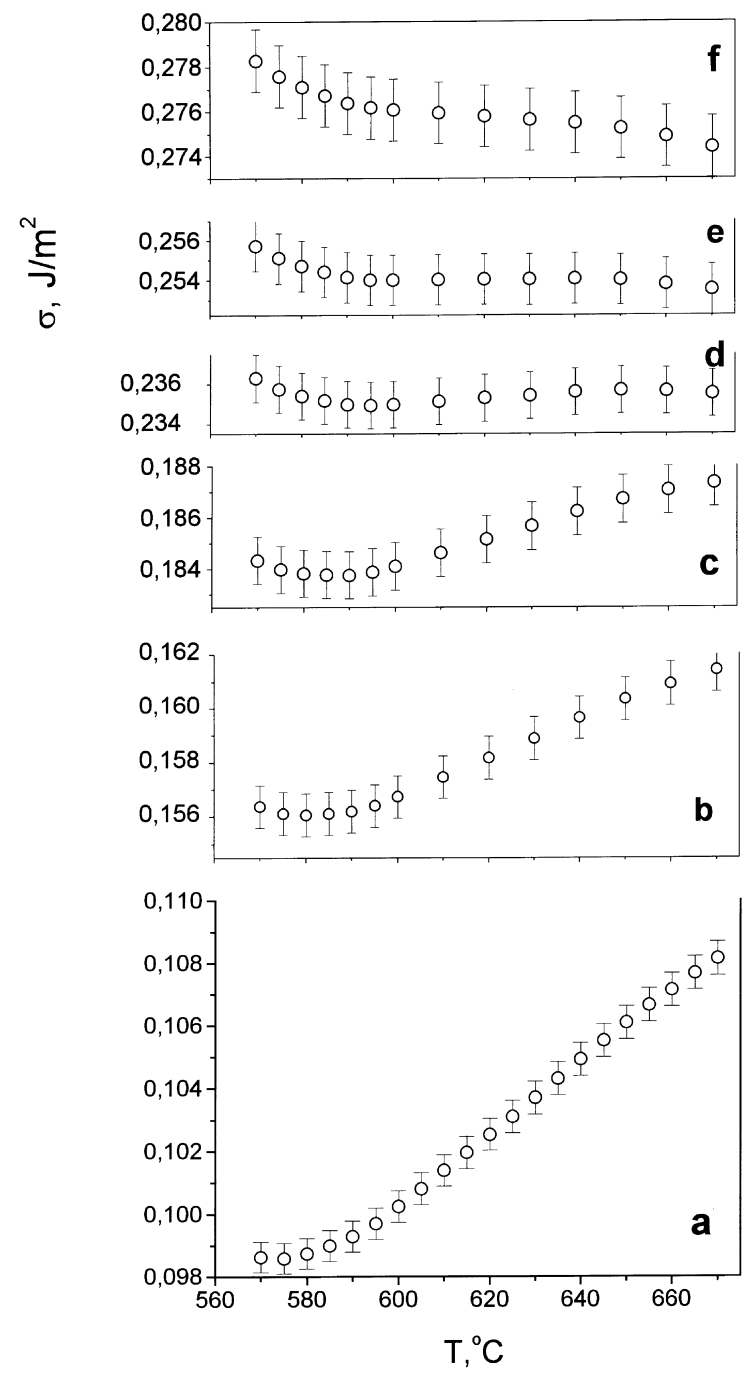

Fig. 4. Crystal/liquid surface energy for N2C3S glass calculated from Eq. (7), with different Tolman's parameters $\delta$ : 0 (a); $2.95 \times 10^{-10}$ (b); $4.5 \times 10^{-10}$ (c); 7.5 (d); $8.65 \times 10^{-10}$ (e) and $10^{-9} \mathrm{~m}$ (f).
The numerical calculations allow one to find the values of surface energy for which the calculated nucleation rates equal the experimental ones (at a given set of Tolman's parameter and driving force). The experimental data were taken from the smoothed $I(T)$ curves. The errors in nucleation
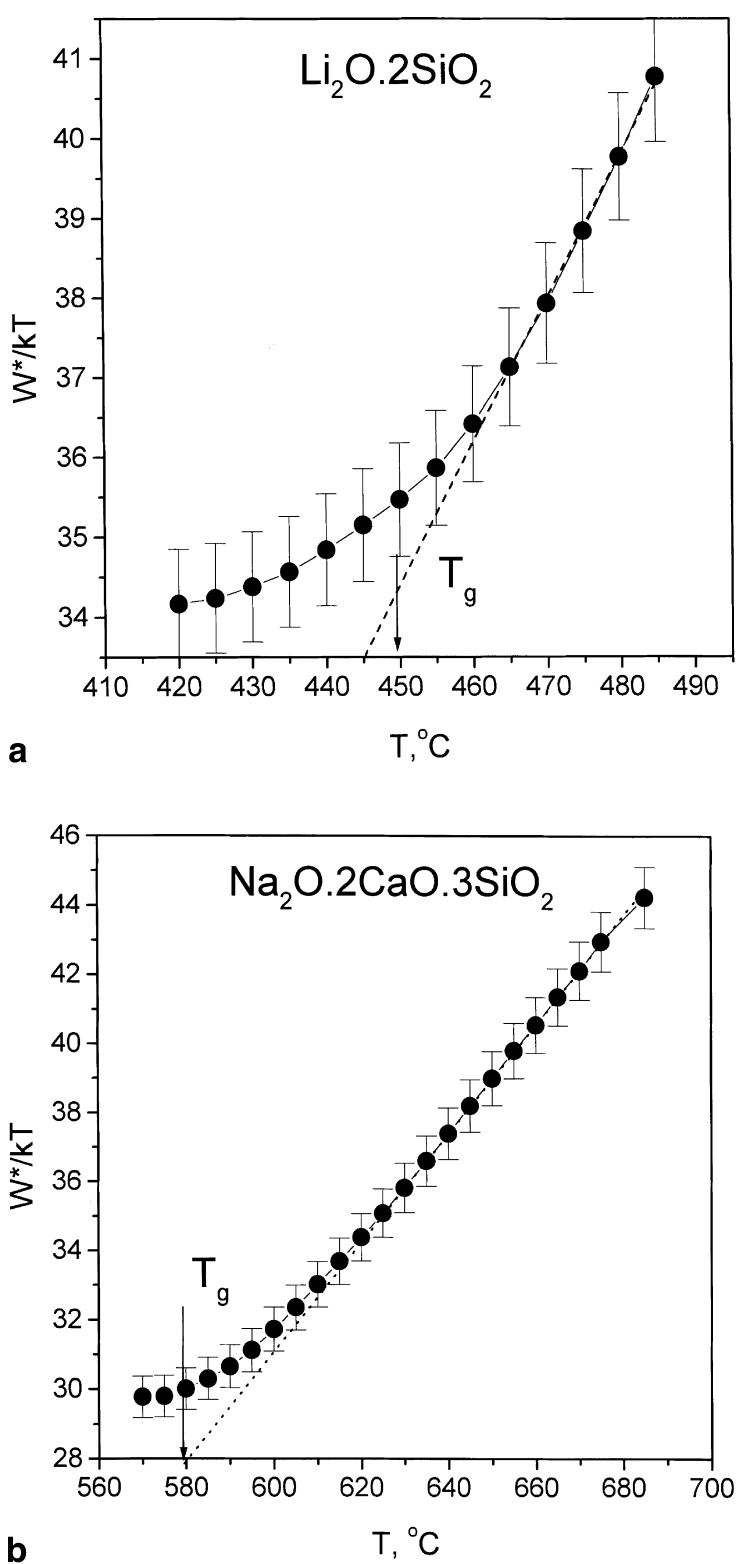

Fig. 5. (a) $W^{*} / k T$ as a function of temperature for LS2 glass. (b) $W^{*} / k T$ as a function of temperature for N2C3S glass. 
rates were about $20 \%$. In this case, $W^{*} / k T$ varies $\sim 2 \%$ and $\sigma \sim 0.5 \%$ (shown in Figs. $3-5$ )

\section{Discussion}

As follows from Figs. 3 and 4, the calculation of $\sigma_{\infty}(T)$ is strongly affected by the choice of Tolman's parameter. The case of $\delta=0$ corresponds to a size independent surface energy, as employed by most authors. In this case, surface energy increases with temperature $\left(\mathrm{d} \sigma_{\infty} / \mathrm{d} T>0\right)$, from the glass transition temperature, $T_{\mathrm{g}}$. At temperatures below $T_{\mathrm{g}}$, surface energy changes very slightly (N2C3S glass) or is reduced to a minimum (L2S glass).

It is possible that, at these low temperatures, the elastic strains resulting from the difference between the densities of crystal and glass are important. In this case, the thermodynamic driving force for crystallization, $\Delta G_{\mathrm{V}}$, would be diminished by the elastic strain energy, which in turn would result in an increase of the thermodynamic barrier to nucleation, $W^{*}$. Fig. 5 gives circumstantial evidence for this assumption, i.e., the decrease of $W^{*}$ slows down at temperatures close to $T_{\mathrm{g}}$. Thus, when one uses overestimated values of $\Delta G_{\mathrm{V}}$ (at low temperatures), one also obtains overestimated values of surface energy. This feature may explain the shape of $\sigma_{\infty}(T)$ dependence at low temperatures.

Fig. 6 shows the average values of $\mathrm{d} \sigma_{\infty} / \mathrm{d} T$ at $T \geqslant T_{\mathrm{g}}$ vs. Tolman's parameter. As $\delta$ increases, $\mathrm{d} \sigma_{\infty} / \mathrm{d} T$ reduces progressively and becomes negative at $\delta>2.6 \times 10^{-10} \mathrm{~m}$ (L2S glass); $8 \times 10^{-10} \mathrm{~m}$ (N2C3S glass). Thus, the Tolman parameter may be chosen so that the surface tension, $\sigma_{\infty}$, increases with decreasing temperature, in line with theory [10].

Moreover, one can estimate $\left(\sigma_{\mathrm{m}}-\sigma\left(T_{\mathrm{g}}\right)\right) /\left(T_{\mathrm{m}}\right.$ $\left.-T_{\mathrm{g}}\right) \cong \mathrm{d} \sigma / \mathrm{d} T$ using Eq. (22) from [10]

$\sigma(T) / \sigma_{\mathrm{m}}=1+(g / \gamma)(\varepsilon-1) n_{\mathrm{i}} \Delta G(T) / \Delta H_{\mathrm{m}}$,

where $\gamma$ is the coefficient in the well-known Scapski-Turnbull equation:

$\sigma_{\mathrm{m}}=\gamma \Delta H_{\mathrm{m}} / v_{\mathrm{c}}^{2 / 3} N_{a}^{1 / 3}$,

where $\varepsilon=v_{\mathrm{f}} / v_{\mathrm{c}}\left(1-g+g v_{\mathrm{f}} / v_{\mathrm{c}}\right), g$ is a numerical factor between 0 and $1, v_{\mathrm{f}}$ and $v_{\mathrm{c}}$ the molar volumes of the melt and crystal, respectively and $n_{\mathrm{i}}$ is
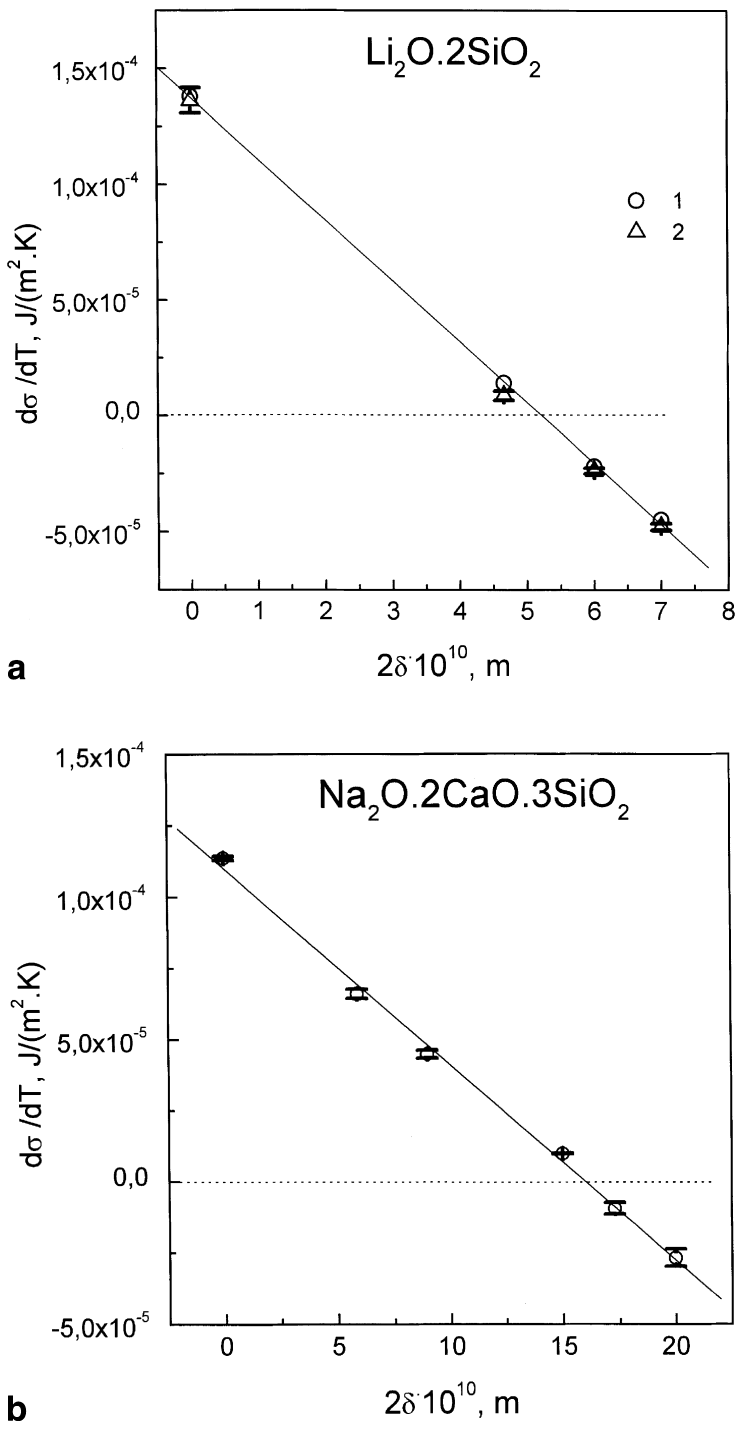

Fig. 6. (a) $d \sigma_{\infty} / d T$ as a function of Tolman's parameter for LS2. $\sigma_{\infty}$ was calculated from Eq. (7) -1 and Eq. (8) - 2. (b) $\mathrm{d} \sigma_{\infty} / \mathrm{d} T$ as a function of Tolman's parameter for N2C3S. $\sigma_{\infty}$ was calculated from.Eq. (7).

the number of monolayers of the interface phase. For $v_{\mathrm{f}} / v_{\mathrm{c}}=1.04(\mathrm{~L} 2 \mathrm{~S}), 1.02(\mathrm{~N} 2 \mathrm{C} 3 \mathrm{~S}), \Delta G\left(T_{\mathrm{g}}\right) /$ $\Delta H_{\mathrm{m}}=0.42(\mathrm{~L} 2 \mathrm{~S}), 0.30(\mathrm{~N} 2 \mathrm{C} 3 \mathrm{~S}), \gamma=0.4, n_{\mathrm{i}}=2-4$ and $g=0.5$, which provides the largest effect, $-\mathrm{d} \sigma_{\infty} / \mathrm{d} T$ varies from $2 \times 10^{-5}$ to $4 \times 10^{-5} \mathrm{~J} \mathrm{~m}^{-2}$ $\mathrm{K}^{-1}$ for L2S glass and from $0.4 \times 10^{-5}$ to $0.7 \times 10^{-5} \mathrm{~J} \mathrm{~m}^{-2} \mathrm{~K}^{-1}$ for N2C3S glass. These results are close to those calculated from the exper- 
imental nucleation rates for $2.8 \times 10^{-10}<$ $\delta<3.4 \times 10^{-10} \mathrm{~m} \quad(\mathrm{~L} 2 \mathrm{~S})$ and $8.2 \times 10^{-10}<$ $\delta<8.5 \times 10^{-10} \mathrm{~m}$ (N2C3S) (Fig. 6).

Although these calculations are tentative, one can conclude that, by using reasonable values for Tolman's parameters, it is possible to obtain the temperature dependence of $\sigma_{\infty}$ predicted by theory. Hence, the increase of the surface energy, $\sigma\left(R^{*}\right)$ with temperature, calculated by fitting nucleation rates to the CNT, is, indeed, caused by a size effect, namely, by the change of the critical size $R^{*}$. Thus, one may reach an agreement between an experiment and the CNT when the curvature dependence of the surface tension is taken into account.

\section{Conclusions}

The crystal/liquid surface energy $\sigma_{\infty}$ was calculated from nucleation rate data for two silicate glasses, using Tolman's equation for size dependent surface tension. Reasonable values for the Tolman parameter may be chosen so that the surface tension decreases with temperature. The temperature dependence of surface energy is close to the theoretical predictions.

\section{Acknowledgements}

We express our appreciation of the funding provided by the Brazilian financing agencies CNPq, PRONEX and FAPESP and our indebtedness to Dr J.W.P. Schmelzer and Professor I. Gutzow for their critical comments.

\section{References}

[1] J.W. Christian, The Theory of Transformation in Metals and Alloys, Part 1, Pergamon, Oxford, 1981.

[2] E.D. Zanotto, P.F. James, J. Non-Cryst. Solids 74 (1985) 373.
[3] P.F. James, J. Non-Cryst. Solids 73 (1985) 517.

[4] D. Turnbull, J. Chem. Phys. 20 (1952) 411.

[5] Y. Miyazawa, G.M. Pound, J. Cryst. Growth 23 (1974) 45.

[6] E.G. Rowlands, P.F. James, Phys. Chem. Glasses 20 (1979) 9.

[7] V.M. Fokin, A.M. Kalinina, V.N. Filipovich, J. Cryst. Growth 52 (1981) 115.

[8] O.V. Potapov, V.M. Fokin, V.N. Filipovich, J. Non-Cryst. Solids 1999, accepted.

[9] A.I. Rusanov, Fazovye Ravnovesiya i Poverkhnostnye Yavlenia, Izd. Khimya, Leningrad, 1967.

[10] I. Gutzow, D. Kashchiev, I. Avramov, J. Non-Cryst. Solids 73 (1985) 477.

[11] D. Turnbull, Thermodynamics and kinetics of formation of the glass state and initial devitrification, in: Physics of Non-Crystalline Solids, North Holland, Amsterdam, 1964, p. 41.

[12] O.V. Mazurin, M.V. Streltsina, T.P. Shvaiko-Shvaikovskaya, Handbook of Glass Data Part A, Elsevier, Amsterdam, 1983.

[13] R. Kaischev, B. Mutaftschiev, Bull. Inst. Chim. Bulg. Acad. Sci. 7 (1959) 177.

[14] M.C. Weinberg, E.D. Zanotto, S. Manrich, Phys. Chem. Glasses 33 (1992) 99.

[15] Z. Kozisek, Cryst. Res. Technol. 26 (1991) 3.

[16] J.W.P. Schmelzer, I. Gutzow, J. Schmelzer Jr., J. Colloid Interface Sci. 178 (1996) 657.

[17] E. Meyer, J. Non-Cryst. Solids 130 (1991) 287.

[18] M. Larson, J. Garside, J. Cryst. Growth 76 (1986) 88.

[19] J.W. Gibbs, Trans. Conn. Acad. 3 (1878) 343.

[20] R.C. Tolman, J. Chem. Phys. 17 (1949) 333.

[21] D.H. Rasmussen, J. Cryst. Growth 56 (1982) 45.

[22] W. Vogelsberger, G. Marx, Z. Phys. Chem. 257 (1976) 580.

[23] J. Schmelzer, R. Mahnke Soc, J. Chem. Soc. Faraday Trans. 82 (1986) 1413.

[24] V.P. Klyev, private communication, 1999.

[25] V.M. Fokin, E.D. Zanotto, J. Non-Cryst. Solids 246 (1999) 115.

[26] A.M. Kalinina, V.N. Filipovich, V.M. Fokin, G.A. Sycheva, Proceedings of the XIVth Congress on Glass ICG 1 (1986) 366.

[27] O.V. Potapov, MSc thesis, St. Petersburg University, 1996.

[28] K. Takahashi, T. Yoshio, Yogyo-Kyokai-Sch 81 (1973) 524.

[29] C.J.R. Gonzalez-Oliver, PhD thesis, University of Sheffield, 1979.

[30] V.I. Babushkin, G.M. Matveyev, O.P. Mchedlov-Petrossyan, Thermodynamics of Silicates, Springer, Berlin, 1985. 